\title{
Microwave Absorbing Properties of Ni/Ferrite Mixture
}

\author{
Liu Xing, Pan Shunkang, Cheng Lichun, Li Chunming, Mo Huihai, Zhou Huaiying
}

Guangxi Key Laboratory of Information Materials, Guilin University of Electronic Science and Technology, Guilin 541004, China

\begin{abstract}
The Sr-W type planar hexagonal ferrite was synthesized by a sol-gel method, and its morphology and phase structure were characterized by X-ray diffraction (XRD) and scanning electron microscopy (SEM). The microwave absorbing properties of the mixtures with different ratios of $\mathrm{Ni} /$ ferrite were studied by vector network analyzer (VAZ). The results demonstrate that with the increase of hexagonal ferrite content, the microwave absorbing peaks of the mixtures shift to higher frequencies, and when the mixture consists of $20 \% \mathrm{Ni}$ and $80 \% \mathrm{Sr}$-W-type ferrite, its microwave absorbing performance is the most advantageous. When absorbing coating thickness is $2 \mathrm{~mm}$, the minimum reflectivity and absorbing peak frequency is $-20.69 \mathrm{~dB}$ at $12.08 \mathrm{GHz}$ and the bandwidth less than $-10 \mathrm{~dB}$ reaches $4 \mathrm{GHz}$. The absorbing peak shifts to low-frequency with the increase of absorbing coating thickness, and when the coating thickness reaches a certain value, the two absorbing peaks appear in the mixture in the frequency range of $2 \sim 18 \mathrm{GHz}$, which is of significance to explore the absorbing material with bandwidth effect.
\end{abstract}

Key words: planar hexagonal ferrite; sol-gel method; Sr-W-type ferrite; absorbing property

With the progress of science and technology, electronic products have entered into all aspects of our lives which bring us so many convenient but at the same time it also leads to a problem of electromagnetic pollution ${ }^{[1-6]}$. Therefore, solving of the problem has become a worldwide issue, and researching and preparing of the absorbing materials that can transform electromagnetic wave energy into other forms of energy are more and more important. At present, ferrite absorbing materials have been widely studied and used, but due to the Snoek restriction of ferrite magnetic materials, traditional ferrite absorbing materials can not been used in the ultra-high frequency ${ }^{[7-10]}$. Compared with the traditional ferrite, plane hexagonal ferrite materials possess high magnetic permeability, large saturated magnetization, high resistance, good stability and magnetocrystalline anisotropy, and they can break though the Snoek restriction to be used in high frequency ${ }^{[11,12]}$. In order to improve the effective bandwidth of material, adding some other types of medium in strontium ferrite forming composite absorbing materials is an effective way. In this paper, Sr-W type ferrite was synthesized by a citric acid sol-gel method and the absorbing properties of $\mathrm{Ni} /$ ferrite mixture in the frequency range of $2 \sim 18 \mathrm{GHz}$ were studied.

\section{Experiment}

Based on the chemical formula $\mathrm{Sr}_{3} \mathrm{Co}_{2} \mathrm{Fe}_{24} \mathrm{O}_{41}$, the analytical reagent grade strontium nitrate, nickel nitrate, and iron nitrate were calculated and weighted, then the weighed chemical reagent was dissolved in distilled water, and the citric acid solution was obtained according to mole ratio of metal ions to citric acid $1: 1$, the metal ions solution and the citric acid solution were mixed together and stirred with a glass rod. Then the mixed solution was adjusted to weak alkaline (the $\mathrm{pH}$ value was 7 8) by ammonia, followed by placing the homogeneous colloidal sol on the top of a hot plate for gelating at $85{ }^{\circ} \mathrm{C}$ with the magnetic stirring bar. The obtained polymerized gel was sintered at $1250{ }^{\circ} \mathrm{C}$ for $5 \mathrm{~h}$. The phase structure and the morphologies of the powders were characterized by XRD (Empyrean PIXcel 3D) and SEM (JSM-5610LV), and then the $\mathrm{Ni} /$ ferrite powders were mixed together with different mass fraction $(0,2040,60,80,100$, $\mathrm{wt} \%)$. For testing the electromagnetic parameters $\left(\varepsilon^{\prime}, \varepsilon^{\prime \prime}, \mu^{\prime}\right.$, $\left.\mu^{\prime \prime}\right), \mathrm{Ni} /$ ferrite mixture was made into a coaxial ring (the

Received date: September 14, 2014

Foundation item: National Natural Science Foundation of China (51361007); National Natural Science Foundation of Guangxi (2012GXNSFGA06002)

Corresponding author: Pan Shunkang, Ph. D., Professor, School of Materials Science and Engineering, Guilin University of Electronic Technology, Guilin 541004, P. R. China, Tel: 0086-773-2291434, E-mail: skpan88@163.com 
thickness, the inner and outside diameters were about 3.5, 3 and $7 \mathrm{~mm}$, respectively) which were measured by a VNA (Agilent8722ES) for calculating the reflectivity of the sample. Table 1 shows the compositions of each sample.

\section{Results and Discussion}

\subsection{Morphology and microstructure of Sr-W-type ferrite}

Fig. 1 is SEM image of the Sr-W-type ferrite prepared by the sol-gel method. As can be seen from the image, the ferrite powders (i.e. absorber) exhibit a good flakiness effect and the average crystal particle size is about $10 \mu \mathrm{m}$. Studies of Ge et al. ${ }^{[13]}$ have found that the microwave absorbing properties of the absorbing material have a close relation with the shape of an absorber, and the acicular or the flakiness is the best shape of the absorber. Therefore, the appearance of the sample implies that it will have a good microwave absorbing performance.

Fig. 2 shows the XRD pattern of the ferrite powder prepared by the sol-gel method. From it can be seen that, the powder mainly consists of $\mathrm{W}$-type phase and a small amount of Z-type phase after being sintered at $1250^{\circ} \mathrm{C}$ for $5 \mathrm{~h}$.

\subsection{Electromagnetic microwave absorbing properties of $\mathrm{Ni} /$ ferrite mixture}

As seen from the dielectric constant figure (Fig.3), as the ferrite content in the sample increases gradually, the values of dielectric constant real part $\varepsilon^{\prime}$ and dielectric constant imaginary part $\varepsilon^{\prime \prime}$ present an increasing trend. When the ferrite content in the sample is less than $40 \%$, the values of $\varepsilon^{\prime}, \varepsilon^{\prime \prime}$ change quite a little in $2 \sim 18 \mathrm{GHz}$, but when it is more than $40 \%$, the values $\varepsilon^{\prime}, \varepsilon^{\prime \prime}$ decrease with the increase of frequency. As ferrite content reaches $100 \%$, the values of $\varepsilon^{\prime}, \varepsilon^{\prime \prime}$ increase first, then peak appear, and finally they decrease with frequency increasing. The cause of the phenomenon appearing above is that with ferrite content increasing, the dielectric properties of the material are

Table 1 Compositions of each samples (wt\%)

\begin{tabular}{ccccccc}
\hline Sample No. & S1\# & S2\# & S3\# & S4\# & S5\# & S6\# \\
\hline Ni & 100 & 80 & 60 & 40 & 20 & 0 \\
Ferrite & 0 & 20 & 40 & 60 & 80 & 100 \\
\hline
\end{tabular}

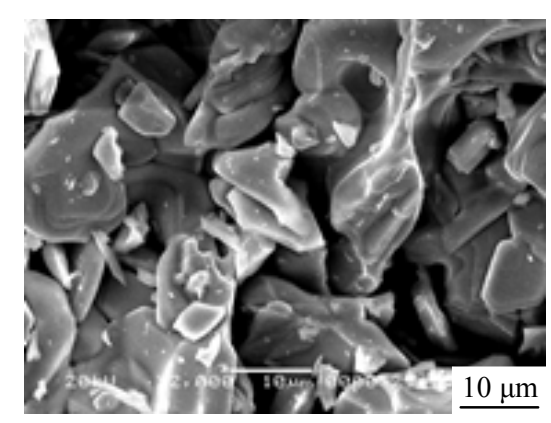

Fig.1 SEM image of the Sr-W-type ferrite prepared by the sol-gel method

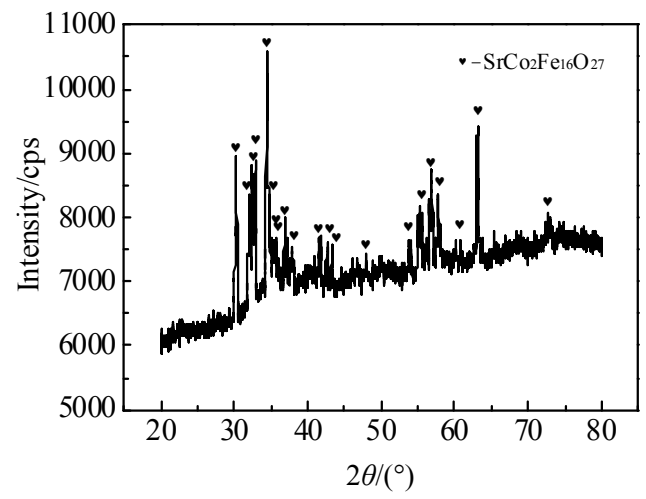

Fig.2 XRD pattern of $\mathrm{SrCo}_{2} \mathrm{Fe}_{16} \mathrm{O}_{27}$ ferrite prepared by the sol-gel method

enhanced constantly, and when the frequency is high enough, the dipole rotation cannot keep the changing pace of the electric field periodic, this polarized form has little contribution to improvement of the coefficient of permittivity and the coefficient variation tend to zero along with the frequency rising; therefore the coefficient of permittivity is provided only by the displacement polarization, and this process will consume the partial energy. In the frequency range of $2 \sim 18 \mathrm{GHz}$, the permeability real part $\mu^{\prime}$ of each sample declines with the increase of frequency and for the permeability imaginary part $\mu$ " natural resonance peaks appear at $5.92,6.48,7.04,10.8,7.12$ and $8 \mathrm{GHz}$.

According to the transmission line theory ${ }^{[14,15]}$, the reflection coefficient for coated single-layer electromagnetic microwave absorbing materials can be deduced as follows:

$$
\begin{aligned}
& R=20 \lg (\mid Z-1) /(Z+1) \mid \\
& Z=\sqrt{\mu_{\mathrm{r}} / \varepsilon_{\mathrm{r}}} \tanh \left(\mathrm{j} 2 \pi f d \sqrt{\mu_{\mathrm{r}} \varepsilon_{\mathrm{r}}} / c\right)
\end{aligned}
$$

where, $\varepsilon_{\mathrm{r}}, \mu_{\mathrm{r}}$ and $d$ are the relative dielectric constant, relative permeability and thickness of the absorbing material, respectively, $f$ is electromagnetic wave frequency, $c$ is electromagnetic wave in free space velocity, and $\mathrm{j}$ denotes imaginary unit. The reflectivity $(R)$ of wave-absorbing materials was calculated using formula (1).

Fig. 4 shows the reflectivities of the different contents of $\mathrm{Ni}$ /ferrite mixture samples with $2 \mathrm{~mm}$ absorbing coating thickness. As can be seen from the figure, with the increase of ferrite content in the mixture, the absorption peak moves to low frequency, the reflectivity values decrease first, and then increase. When absorbing coating thickness $d=2 \mathrm{~mm}$, the minimum absorption reflectivity peak frequency of S6\#, S5\#, S4\#, S3\#, S2\#, S1\# were $-2.98 \mathrm{~dB}$ at $2.88 \mathrm{GHz},-4.38 \mathrm{~dB}$ at $3.68 \mathrm{GHz},-7.92 \mathrm{~dB}$ at $4.8 \mathrm{GHz},-17.48 \mathrm{~dB}$ at $7.6 \mathrm{GHz}$, $-20.69 \mathrm{~dB}$ at $12.08 \mathrm{GHz}$ and $-11.45 \mathrm{~dB}$ at $15.04 \mathrm{GHz}$, respectively. Sample S2\# has minimum absorption reflectivity value, and the bandwidth is reasonable, so in this experiment, $80 \% \mathrm{Ni}$ and $20 \% \mathrm{Sr}-\mathrm{W}$ type ferrite mixture samples exhibits the best microwave absorbing performance. 

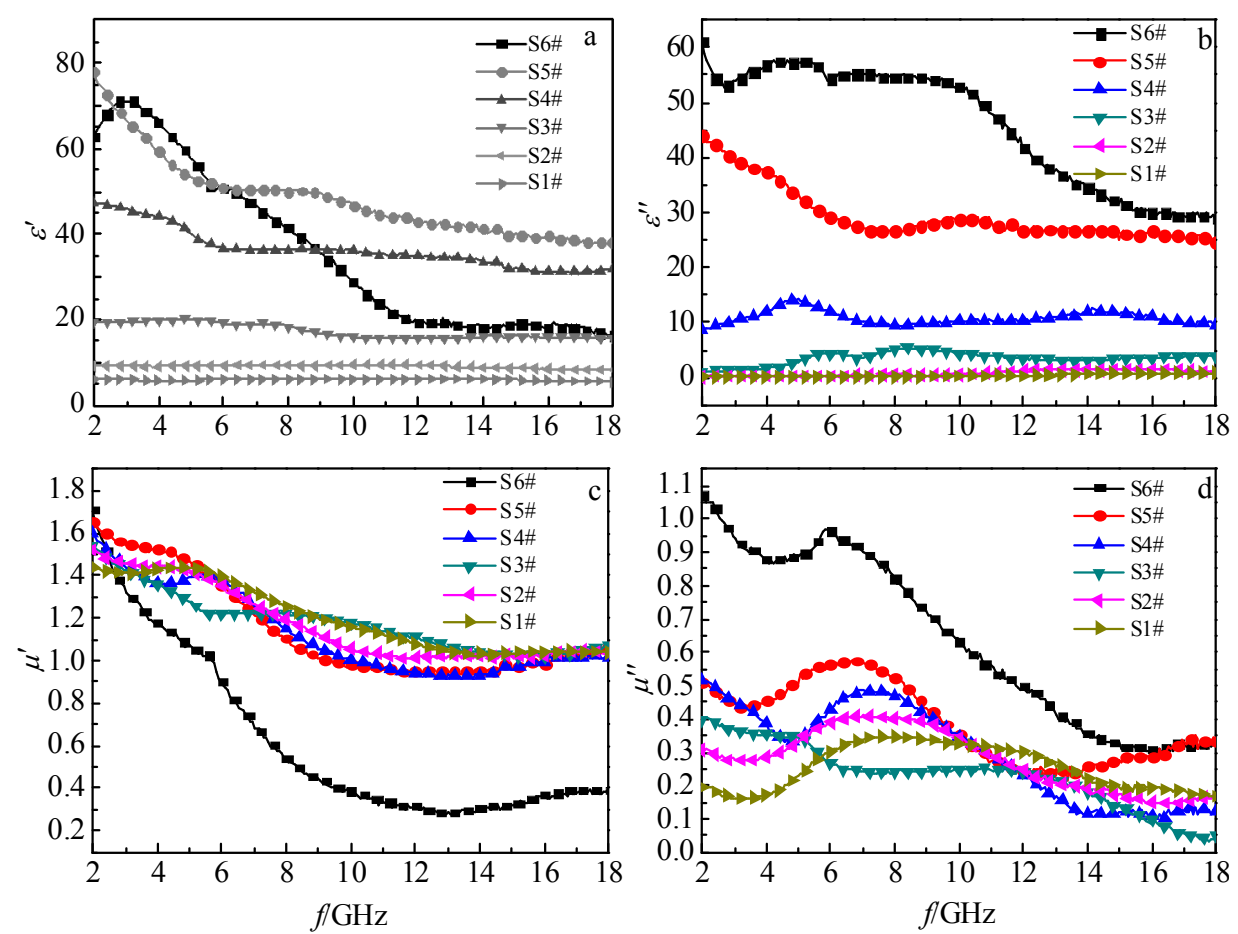

Fig.3 Electromagnetism parameters of powders in the frequency range of $2 \sim 18 \mathrm{GHz}$ : (a) $\varepsilon^{\prime}$ and (b) $\varepsilon^{\prime \prime}$ vs $f$; (c) $\mu^{\prime}$ and (c) $\mu^{\prime \prime}$ vs $f$

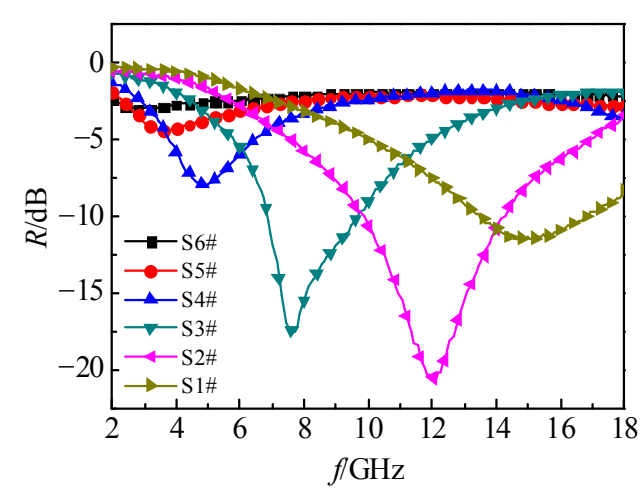

Fig.4 Reflectivity $(R)$ of $\mathrm{Ni} /$ ferrite mixture samples with $2 \mathrm{~mm}$ absorbing coating thickness

Fig. 5 is the reflectivity of sample S4\# in the frequency range $2 \sim 18 \mathrm{GHz}$ with different absorbing coating thicknesses. From the curve we can see that when absorbing coating thickness changes from $1 \mathrm{~mm}$ to $2 \mathrm{~mm}$, the reflectivity decreases gradually and the absorption peak frequency moves towards low frequency. When absorbing coating thickness $d=$ $2 \mathrm{~mm}$, the minimum reflectivity and absorbing peak frequency is $-20.69 \mathrm{~dB}$ at $12.08 \mathrm{GHz}$, and the band width less than -10 $\mathrm{dB}$ reaches $4 \mathrm{GHz}$. When the coating thickness continues to increase, the reflectivity increases and absorption peak frequency continues to move towards low frequency, and the band width decreases. When the thickness increases to more than $5 \mathrm{~mm}$, two absorption peaks appear in the sample, and the distance between the two absorption peaks has a tendency

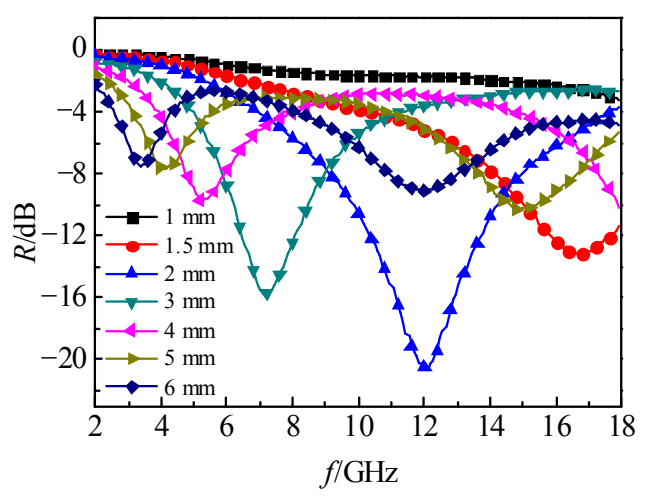

Fig.5 Reflectivity $(R)$ of sample S4\# with different absorbing coating thickness

to decrease with the thickness increasing, and the phenomenon is very interesting to the broadband absorbing material.

\section{Conclusions}

1) With the hexagonal ferrite content decreasing, the microwave absorbing peak of the mixture moves to low frequency, and when the mixture consists of $20 \% \mathrm{Ni}$ and $80 \%$ Sr-W type ferrite, the microwave absorbing performance is the most advantageous, the minimum reflectivity and absorbing peak frequency is $-20.69 \mathrm{~dB}$ at $12.08 \mathrm{GHz}$ and the bandwidth less than $-10 \mathrm{~dB}$ up to $4 \mathrm{GHz}$.

2) The absorbing peak shifts to low-frequency with the absorbing coating thickness increasing and when the coating thickness reaches a certain value, two absorbing peaks appear 
in the mixture in the frequency range $2 \sim 18 \mathrm{GHz}$.

\section{References}

1 Magali Silveira Pinhoa, Maria Luisa Gregoria, Regina Célia Reis Nunes et al. European Polymer Journal[J], 2002, 38(11): 2321

$2 \mathrm{Gu}$ Xiaocheng, Liang Fengying. World Environment $[\mathrm{J}]$, 2013(01): 84

3 Zheng Yuling, Yu Jianjun, Qin Jingliang et al. Occup and Health[J], 2011, 27(6): 689

4 Zhao Feng. Urban Environment \& Urban Ecology[J], 2011, 24(5): 39

5 Cui Benliang. Modern Electronics Technique[J], 2011, 34(20): 140

6 Wang Yi. Municipal Administration \& Technology[J], 2001(3): 14

7 Zhang Hongguo, Li Longtu, Zhou Ji et al. IEEE International Symposium on Appl Ferroelectr[J], 2000, 2: 859
8 Pullarl R C, Bhattacharya A K. Materials Research Bulletin[J], 2001, 36: 1533

9 Li Haifeng, Gong Rongzhou, Fan Liren et al. Rare Metal Materials and Engineering[J], 2009, 38(11): 2053 (in Chinese)

10 Dishovski N, Petkov A, Nedkov Iv et al. IEEE Trans Magn[J], 1994, 30: 969

11 Wang Jing, Zhang Hong, Bai Shuxin et al. Journal of Magnetism and Magnetic Materials[J], 2007, 312: 310

$12 \mathrm{Wu}$ Xiaojun, Jing Hongxia, Zhang Cunrui et al. Rare Metal Materials and Engineering[J], 2010, 39(4): 727 (in Chinese)

13 Ge Fuding et al. Aerospace Materials \& Technology[J], 1996(5): 42

14 Naito Y, Suetake K. IEEE Trans Microwave Theory Tech[J], 1971, 19(1): 65

15 Naito Y, Seki H. Electron Commun Jpn Part I[J], 1990, 73(1): 86

\title{
镍粉/平面六角锶铁氧体复合材料微波吸收性能的研究
}

刘 星, 潘顺康, 成丽春, 李春明, 莫辉海, 周怀营

(桂林电子科技大学 广西信息材料重点实验室, 广西 桂林 541004)

\begin{abstract}
摘 要: 采用溶胶凝胶法制备了平面六角铁氧体, 并用扫描电镜 (SEM), X射线衍射仪 (XRD) 对它的形貌以及相结构进行了表征, 用矢 量网络分析仪 (VAZ) 研究了不同比例的 $\mathrm{Ni} /$ 铁氧体混合物的微波吸收性能。结果发现, 随着混合物中的平面六角铁氧体含量增多, 混合 物的微波吸波吸收峰向高频方向移动, 当混合物由 $20 \% \mathrm{Ni}+80 \%$ 铁氧体组成时, 混合物的微波吸收性能最佳。当吸波涂层厚度为 $2 \mathrm{~mm}$ 时, 在 $12 \mathrm{GHz}$ 处, 最小反射率为 $-20 \mathrm{~dB}$, 小于 $-10 \mathrm{~dB}$ 的带宽达到了 $4 \mathrm{GHz}$ 。当吸波涂层厚度增加时, 吸收峰频率向低频移动, 吸收峰值 先变小, 后增大。当厚度达到一定值时, 混合物在 $2 \sim 18 \mathrm{GHz}$ 之间出现 2 个吸收峰, 这对于研究宽频吸收具有重要意义。

关键词: 平面六角铁氧体; 溶胶凝胶法; $\mathrm{W}$ 型锶铁氧体; 微波吸波性能
\end{abstract}

作者简介: 刘 星, 男, 1987 年生, 硕士, 桂林电子科技大学材料科学与工程学院, 广西 桂林 541004, 电话: 0773-2291434, E-mail: liuxing1987q@163.com 